\title{
SCANNING ION-CONDUCTANCE MICROSCOPY METHODS FOR STUDYING LOCAL MECHANICAL PROPERTIES OF LIVING CELLS
}

Aleksei Iakovlev ${ }^{1}$, Nikita Savin ${ }^{2}$, Oleg Suchalko ${ }^{1}$, Vasilii Kolmogorov ${ }^{1}$, Peter Gorelkin ${ }^{3}$, Alexander Erofeev $^{3}$ and Pavel Novak ${ }^{4}$

${ }^{1}$ National University of Science and Technology «MISiS», Moscow, Russia, Moskva, Russia, ${ }^{2}$ National University of Science and Technology "MISIS", Moscow, Russia, ${ }^{3}$ National University of Science and Technology «MISiS», Moscow, Russia, Russia, ${ }^{4}$ National University of Science and Technology «MISiS», Moscow, Russia, United States

Studying of mechanical properties of living cells is crucial due to number of abnormalities, related to mechanical modifications of cell-matrix, e.g. cancer cell metastasis. It is assumed that microscopic changes in cellular mechanics could destabilize the regulation of molecular mechanism of mechanotransduction - intracellular process of sensing mechanical signals and transforming them into chemical responses. Studying this mechanism will lead to the new treatment methods for a large number of disorders.

Atomic force microscopy, as widely used technique, uses direct mechanical contact between the probe and the sample, which could damage or modify it surface, leading to unreliable mechanical data. Scanning ion-conductance microscopy (SICM) is a novel method for qualitative and quantitative mapping. SICM uses an electrolytic current through a probe (nanopipette) as a measure for pipette-surface distance. It detects the ion flow and prevents physical contact of pipette-tip with the sample, and allows to scan live cells in sub-micrometer resolution (Rheinlaender, 2013).

SICM uses two potentially different topography mapping methods. The first one applies constant pressure to the upper end of the pipette with tip radius more than $120 \mathrm{~nm}$. This allows the unhindered pressure supply on the sample. With pipette approaching the pressure on the sample increases and ion current drops (Pellegrino et al., 2012). When the ion current drops more than the first threshold level, the vertical pipette position is stored as a measure of sample height. After reaching a second threshold level the z-piezo drag away the pipette from the sample to avoid mechanical contact. Pressure is applied with our self-invented precious differential system, allowing to apply both positive and negative pressure. Moreover, with this system studying of ion channel transport of living cells could be carried out automatically. Accuracy of the system is supported by PI-controller based on AVR microcontroller. Software for the system was written on Objective Pascal. Standard deviation of applied pressure is less than $50 \mathrm{~Pa}$.

The resulting deformation of an elastic sample calculates as a function of the vertical pipette position. Second one uses nanopipettes with tip radius up to $50 \mathrm{~nm}$. Hydrostatic pressure is not applied, and the stress on the sample is completely due to the intrinsic colloidal pressure between the cell surface and the pipette tip face (Clarke et al., 2016). The stiffness calculated the same way as in the first method. Theoretical model of both methods is the same, but no one have ever compared them.

As a result, two methods were compared. They yield the same mechanical properties: $887.41 \pm 178.31$

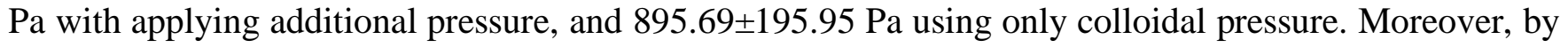
applying colloidal pressure, we could study cell-surface macromolecules. While with hydrostatic pressure, mechanical data consists only of cell membrane stiffness without any macromolecules. 
This research was funded by Ministry of Education and Science of the Russian Federation implemented by a governmental decree, The Russian Science Foundation, grant number №19-79-30062.
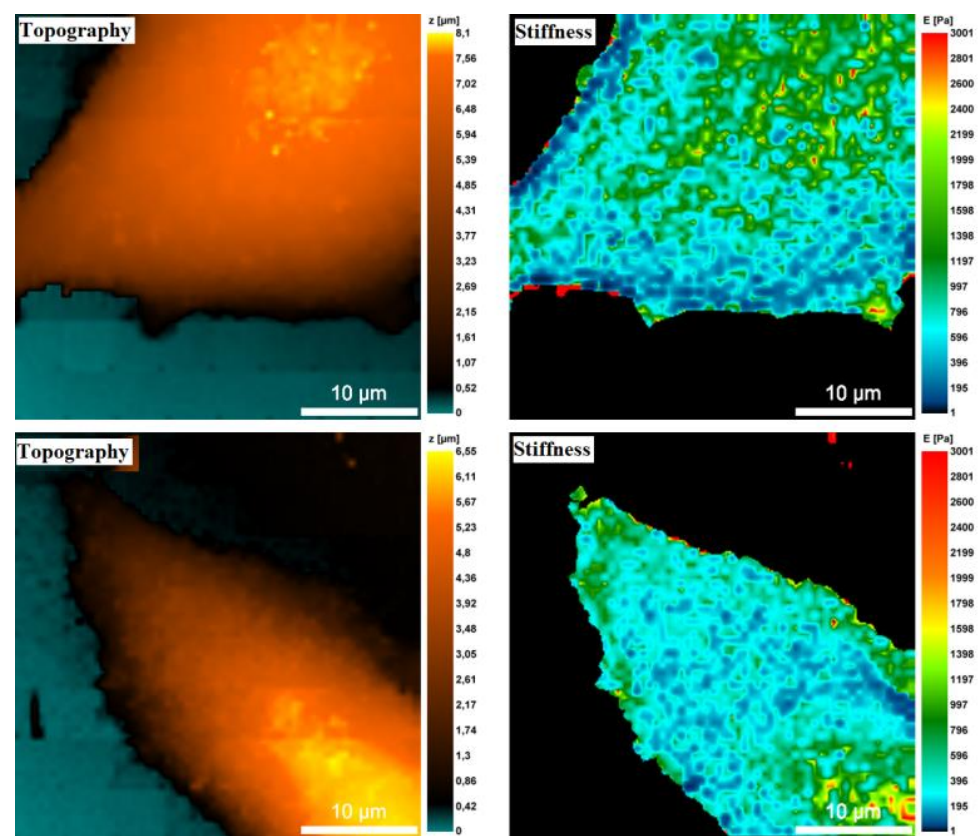

Figure 1. Topography and cell stiffness measured with applying hydrostatic pressure.
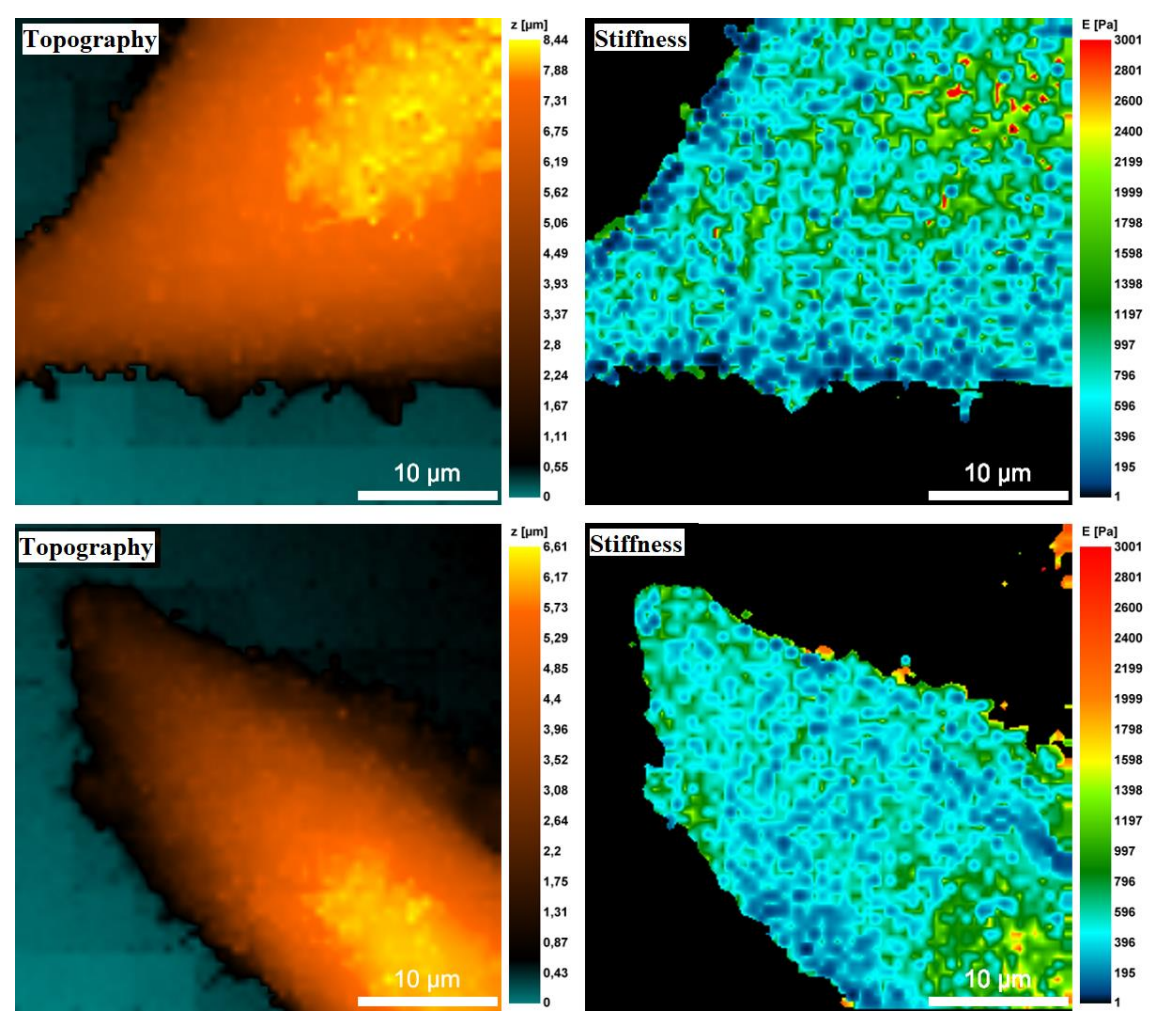

Figure 2. Topography and cell stiffness measured with colloidal intrinsic pressure.

\section{References}


1)Rheinlaender, J. \& Schäffer, E. (2013). Mapping the mechanical stiffness of live cells with the scanning ion conductance microscope. Soft Matter 9, 3230.

2) Pellegrino, M., Pelligrini, M., Orsini, P., Tognoni, E., Ascoli, C., Baschieri, P., \& Dinelli, F. (2012). Measuring the elastic properties of living cells through the analysis of current-displacement curves in scanning ion conductance microscopy. Pflugers Archiv European Journal of Physiology, 464(3), 307-316. 3) Clarke, R., Novak, P., Zhukov, A., Tyler, E., Cano-Jaimez, M., Drews, A., Richards, O., Volynski, K., Bishop, C. \& Klenerman, D. (2016). Low Stress Ion Conductance Microscopy of Sub-Cellular Stiffness. Soft Matter, 12 (38), 7953-7958 